\title{
降冰片烯荧光三唑化合物的合成及其金属离子识别
}

\author{
祝文斌吕小兰朱江华曹迁永* \\ (南昌大学化学学院 鄱阳湖环境与资源利用教育部重点实验室 南昌 330031)
}

\begin{abstract}
摘要 通过点击反应合成了一种以降冰片烯为骨架含葱荧光团环状双三唑化合物 1 及其非环对应物 $\mathbf{2}$, 并研究了其对 金属离子苂光识别能力. 在 $\mathrm{CH}_{3} \mathrm{CN}-\mathrm{H}_{2} \mathrm{O}(\mathrm{V}: V=90: 10)$ 溶液中, 受体分子 1 仅对 $\mathrm{Hg}^{2+}$ 有很好的荧光淬灭响应. 有意思 的是, 非环受体 2 对 $\mathrm{Zn}^{2+}, \mathrm{Hg}^{2+}$ 与 $\mathrm{Cu}^{2+}$ 都有苂光识别响应, 其中滴加 $\mathrm{Zn}^{2+}$ 使受体 $\mathbf{2}$ 苂光增强, 而滴加 $\mathrm{Hg}^{2+}$ 与 $\mathrm{Cu}^{2+}$ 则导 致其苂光淬灭. 同时对受体分子与金属离子的结合模式通过 ${ }^{1} \mathrm{H}$ NMR 滴定及密度泛函理论(DFT)计算进行了探讨.

关键词 降冰片烯; 葱; 三坐; 荧光; 金属离子识别
\end{abstract}

\section{Norbornene-Based Triazoles for Fluorescent Sensing of Metal Ions}

\author{
Zhu, Wenbin Lü, Xiaolan Zhu, Jianghua Cao, Qianyong* \\ (Department of Chemistry, Nanchang University, Nanchang 330031)
}

\begin{abstract}
A new macrocyclic norbornene-based bis-triazole (1) containing anthracene fluorophore and its acyclic analog 2 have been designed and synthesized by "click reaction", and their fluorescence recognition abilities towards various metal ions have been investigated. In $\mathrm{CH}_{3} \mathrm{CN}-\mathrm{H}_{2} \mathrm{O}(\mathrm{V}: V=90: 10)$ solution, cyclic receptor 1 shows excusive turn-off fluorescent sensing of $\mathrm{Zn}^{2+}$. However, acyclic receptor 2 exhibits fluorescence changes towards $\mathrm{Zn}^{2+}, \mathrm{Hg}^{2+}$ and $\mathrm{Cu}^{2+}$. Upon addition $\mathrm{Zn}^{2+}$, the fluorescence of $\mathbf{2}$ give a large enhancement, while the addition of $\mathrm{Hg}^{2+}$ and $\mathrm{Cu}^{2+}$ leads a fluorescence quenching effect. In addition, the binding mechanisms between receptors and metal ions have been discussed via ${ }^{1} \mathrm{H}$ NMR titration and density functional theory (DFT) calculation technique.
\end{abstract}

Keywords norbornene; anthracene; triazole; fluorescence; metal ions recognition

荧光识别过渡金属及重金属离子化学传感器的设 计与合成, 由于其操作简便, 灵敏度高, 检测速度快等 优点, 近年来引起了广泛的关注 ${ }^{[1 \sim 4]}$. 其中, $\mathrm{Zn}^{2+}$ 与 $\mathrm{Cu}^{2+}$ 是人体必需的微量元素, 参与人体的新陈代谢与多 种酶的合成. 而 $\mathrm{Hg}^{2+}$ 为对人体有毒的一种重金属离子, 会导致消化系统、神经系统、心血管等一系列疾病; 铜 离子摄入过量会导致铜中毒, 严重的导致肝脏疾病甚至 死亡 ${ }^{[57]}$. 因此, 对这些金属离子快速灵敏识别与检测 具有重要的意义.

金属离子苂光化学传感器是指将荧光信号单元及 金属离子识别单元通过共价键键合的功能分子. 其中荧 光信号单元包括稠环芳烃、罗丹明、丹酰氯、荥酰亚胺、 苂光素等, 而识别基团则为 $\mathrm{O} 、 \mathrm{~S} 、 \mathrm{~N}$ 等杂原子. 目前, 基 于光致电子转移(PET)、荧光共振能量转移(FRET)、分 子内电荷转移(ICT)及单分子聚激态发射转换的金属离
子化学传感器已有大量文献报道 ${ }^{[8 ~ 13]}$.

2001 年 Sharpless 等 ${ }^{[14,15]}$ 提出的 $\mathrm{Cu}(\mathrm{I})$ 催化的炔与叠 氮化合物 1,3-偶极加成合成 1,2,3-环三唑化合物, 即 “点 击反应” ，由于其反应条件温和、操作简单、反应产率 高等优点在有机材料、生物及催化领域有着广泛应用. 同时，“点击反应”生成的环三唑不仅是一个共价键间 隔基，同时是金属离子与阴离子的识别单元：其 3-位 $\mathrm{N}$ 原子是金属离子的配位点，而 5-位 $\mathrm{CH}$ 能与阴离子发生 氢键作用，可用于设计合成不同阴/阳离子荧光化学传 感器 ${ }^{[16 ~ 25]}$. 笔者近期关注三唑化合物化学传感器的设 计与合成. 继续前期工作, 本文报道了以降冰片烯为骨 架含葱荧光团环状双三唑化合物 1 及其非环对应物 2, 对其金属离子识别性质通过苂光、 ${ }^{1} \mathrm{H}$ NMR 及 DFT 计算 进行了详细研究.

\footnotetext{
* Corresponding author. E-mail: cqyong@ncu.edu.cn

Received November 2, 2016; revised December 12, 2016; published online December 21, 2016.

Project supported by the National Natural Science Foundation of China (No. 21462027).

国家自然科学基金(No. 21462027)资助项目.
} 


\section{1 结果与讨论}

\section{1 合成目标化合物研究}

目标化合物 1 和 $\mathbf{2}$ 的合成见 Scheme 1. 以葱及降冰 片烯二甲酸䣶为起始原料, 首先根据文献方法 ${ }^{[26 ~ 29]}$ 合 成降冰片烯双炔化合物 3、9,10-二迭氮甲基葱化合物 4 及 9-叠氮甲基葱化合物 5. 非环双三唑化合物 2 以典型 的 “点击反应”催化剂 $\mathrm{CuSO}_{4} / \mathrm{NaVc}$ 催化在 $N, N$-二甲基 甲酰胺(DMF)溶剂中合成, 而环双三坐化受体 $\mathbf{1}$ 以 $1,8-$ 二氮杂二环 [5.4.0]十一碳-7-烯(DBU)/CuI 为催化剂在甲 苯溶液中采用极度稀释法 ${ }^{[21,30]}$ 合成. 其中环受体分子 $\mathbf{1}$ 的收率为 $39 \%$, 而非环受体分子 $\mathbf{2}$ 的收率为 $80 \%$, 与文 献报道值相一致. 同时研究还发现, 非环受体 $\mathbf{2}$ 在大部 分有机溶剂中, 如 $\mathrm{CH}_{2} \mathrm{Cl}_{2}, \mathrm{CHCl}_{3}, \mathrm{CH}_{3} \mathrm{CN}, \mathrm{EtOAc}$, $\mathrm{MeOH}, \mathrm{DMF}$ 及二甲基亚砜(DMSO)中都有很好的溶解 性, 而环受体仅在强极性溶剂 DMF 及 DMSO 中才有好 的溶解性. 它们的结构通过了 ${ }^{1} \mathrm{H}$ NMR, ${ }^{13} \mathrm{C} \mathrm{NMR}$, ESI-TOF-MS 及元素分析进行了表征.

\section{2 阳离子的苂光光谱研究}

首先通过荧光光谱研究了受体分子 $\mathbf{1}$ 与 2 对不同金 属离子的识别能力. 在 $\mathrm{CH}_{3} \mathrm{CN}-\mathrm{H}_{2} \mathrm{O}(V: V=90: 10)$ 溶 液中, 以 $360 \mathrm{~nm}$ 为激发波长, 受体分子 $\mathbf{1}$ 与 $\mathbf{2}$ 在具有基 于葱苂光团强的单体发射峰, 其中 $\mathbf{1}$ 的发射峰处于 400 , 423 和 $449 \mathrm{~nm}$, 而受体分子 2 的发射峰位于于 394,416 和 $439 \mathrm{~nm}$. 受体分子 $\mathbf{1}$ 较 $\mathbf{2}$ 发射峰红移可能归因于 1 中 两个缺电子三唑环对一个葱生色团的强的吸电子效应,
而在受体 2 中则为一个三唑环对一个葱环的吸电子效 应. 当滴加不同金属离子 $\left(\mathrm{Na}^{+}, \mathrm{Mg}^{2+}, \mathrm{Ba}^{2+}, \mathrm{Hg}^{2+}, \mathrm{Cu}^{2+}\right.$, $\left.\mathrm{Cr}^{3+}, \mathrm{Zn}^{2+}, \mathrm{Fe}^{2+}, \mathrm{Ni}^{2+}, \mathrm{Co}^{2+}, \mathrm{Cd}^{2+}, \mathrm{Ag}^{+}\right)$于受体 1 或 2 时, 环状受体分子 1 仅对 $\mathrm{Hg}^{2+}$ 有很好的苂光淬灭响应, 而非 环受体分子 2 对 $\mathrm{Zn}^{2+}, \mathrm{Hg}^{2+}, \mathrm{Cu}^{2+}$ 都表现出不同的荧光 信号响应(图 1). 其中滴加 $\mathrm{Zn}^{2+}$ 时使受体分子的荧光强 度增大, 而滴加 $\mathrm{Hg}^{2+}$ 和 $\mathrm{Cu}^{2+}$ 时导致荧光淬灭响应. 非环 受体 2 差的金属离子选择性可能归因为其柔性可变的双 足构型，可容纳不同离子半径的金属离子配位.

图 2 为受体分子 1 中滴加不同当量 $\mathrm{Hg}^{2+}$ 时荧光光谱 变化图. 随着 $\mathrm{Hg}^{2+}$ 浓度增加, 化合物 1 基于葱单分子发 射峰强度逐渐减弱. 当滴加约 250 equiv. 的 $\mathrm{Hg}^{2+}$ 时, 其 光谱强度变化达到饱和, 其荧光淬灭率 $\left(I-I_{0}\right) / I_{0}$ 达到 $89 \%$. 滴加 $\mathrm{Hg}^{2+}$ 导致 1 荧光减弱可归因于金属离子 $\mathrm{Hg}^{2+}$ 到葱苂光团 PET 淬灭过程, 如同一些文献报道那 样 ${ }^{[11]}$. 根据苂光光谱滴定数据, 根据 Benesi-Hildebrand ${ }^{[31]}$ 方程线性拟合, 可得出 $\mathbf{1}$ 和 $\mathrm{Hg}^{2+}$ 络合化学计量 比为 $1: 1$. 同时算得 $1-\mathrm{Hg}^{2+}$ 的络合常数为 $2.03 \times 10^{3}$ $\mathrm{L} \cdot \mathrm{mol}^{-1}(R=0.998)$, 对 $\mathrm{Hg}^{2+}$ 检测限为 $3.52 \times 10^{-4} \mathrm{~mol} / \mathrm{L}$ ( $R=0.989)$.

本文同时研究了受体 2 中分别滴加 $\mathrm{Zn}^{2+}, \mathrm{Cu}^{2+}$ 与 $\mathrm{Hg}^{2+}$ 时荧光光谱进行测定(图 3), 其一些络合参数列于 表 1. 2 可与 $\mathrm{Zn}^{2+}, \mathrm{Cu}^{2+}$ 与 $\mathrm{Hg}^{2+}$ 形成稳定的 1:1 型络合 物, 可通过苂光 Job 曲线证实. 从图 3 及表 1 的数据可 以发现, 2 与 $\mathrm{Zn}^{2+}, \mathrm{Cu}^{2+}$ 与 $\mathrm{Hg}^{2+}$ 的络合常数分别为

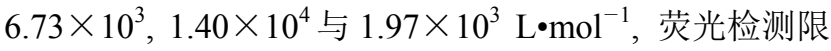

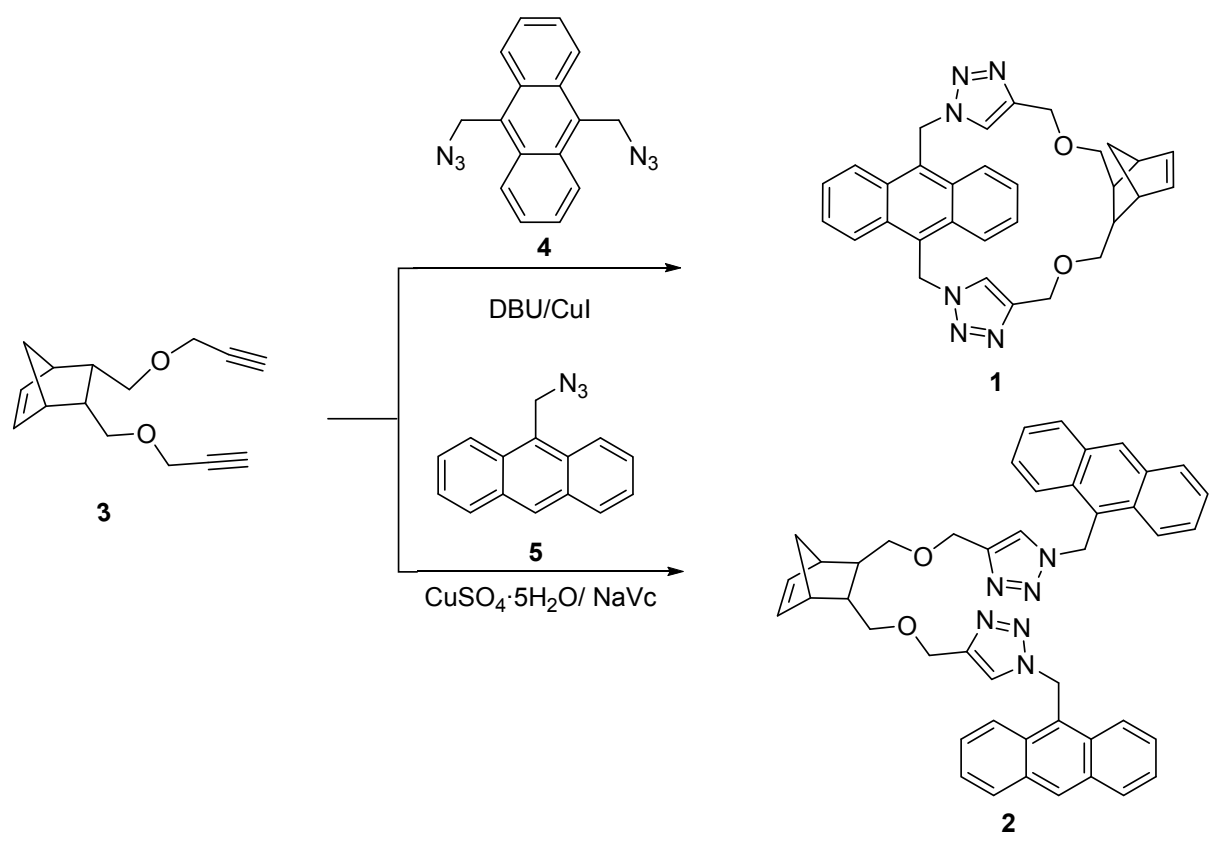

图式 1 目标分子 1 和 $\mathbf{2}$ 的合成路线

Scheme 1 Synthetic route of 1 and 2 

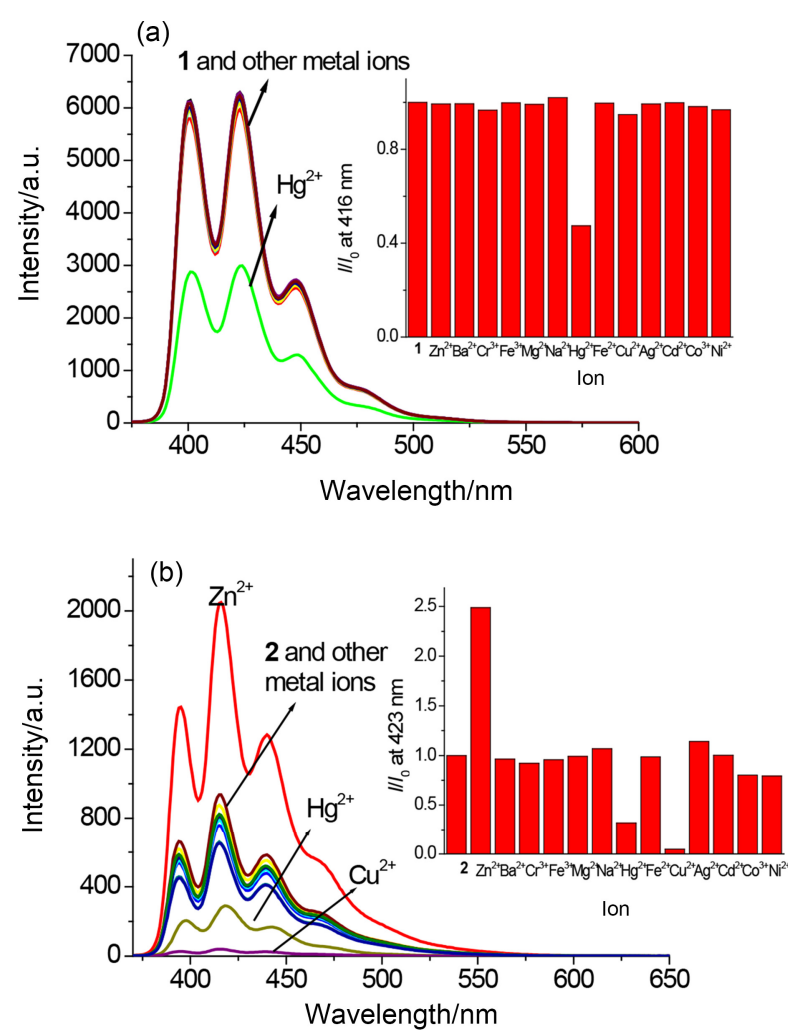

图 1 在 $\mathrm{CH}_{3} \mathrm{CN}-\mathrm{H}_{2} \mathrm{O}(\mathrm{V}: V=90: 10)$ 溶液中化合物 $\mathbf{1}(\mathrm{a})$ 与 2 (b)中滴加等量 (1 为 20 equiv., 2 为 5 equiv.)不同金属离子苂光 光谱变化图

Figure 1 Fluorescence spectra of $\mathbf{1}$ (a) and 2 (b) upon addition equiv. (20 equiv. for 1 and 5 equiv. for 2) of metal ions in $\mathrm{CH}_{3} \mathrm{CN}-\mathrm{H}_{2} \mathrm{O}(V: V=90: 10)$ solution

$c=2.0 \times 10^{-5} \mathrm{~mol} / \mathrm{L}, \lambda_{\text {ex }}=360 \mathrm{~nm}$. Inset: the bar graph of 1 at $423 \mathrm{~nm}$ or 2 at $415 \mathrm{~nm}$ upon addition of various metal ions

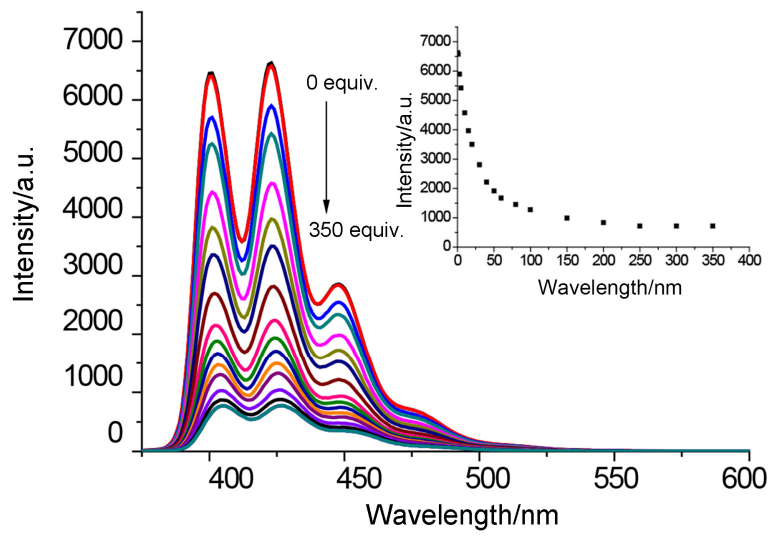

图 2 化合物 $1\left(2 \times 10^{-5} \mathrm{~mol} / \mathrm{L}\right)$ 在 $\mathrm{CH}_{3} \mathrm{CN}-\mathrm{H}_{2} \mathrm{O}(V: V=90$ : 10)中滴加不同当量的 $\mathrm{Hg}^{2+}$ 的苂光光谱图

Figure 2 Fluorescence spectra of $1\left(2.0 \times 10^{-5} \mathrm{~mol} / \mathrm{L}\right)$ upon addition various amount of metal ions in $\mathrm{CH}_{3} \mathrm{CN}-\mathrm{H}_{2} \mathrm{O}(\mathrm{V}: V=$ $90: 10)$ solution

Inset: the bar graph of $\mathbf{1}$ at $423 \mathrm{~nm}$ upon addition various amount of mercury metal ions

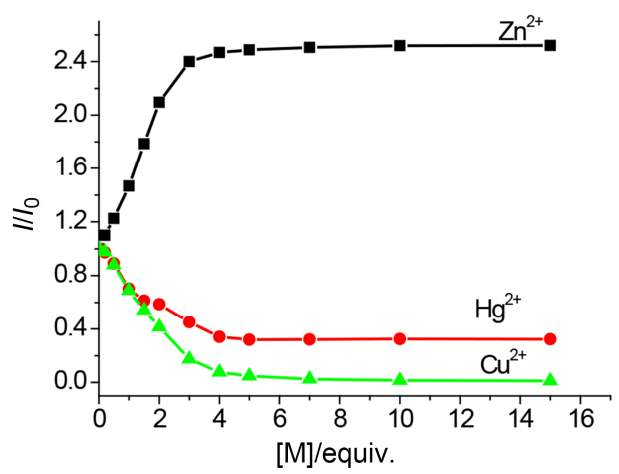

图 $3 \mathrm{CH}_{3} \mathrm{CN}-\mathrm{H}_{2} \mathrm{O}(V: V=90: 10)$ 中化合物 $2\left(2 \times 10^{-5}\right.$ $\mathrm{mol} / \mathrm{L}$ )滴加不同当量的 $\mathrm{Zn}^{2+}, \mathrm{Hg}^{2+}$ 和 $\mathrm{Cu}^{2+}$ 时位于 $416 \mathrm{~nm}$ 荧光 光谱相对强度变化图

Figure 3 Relative fluorescence spectra of $2\left(2.0 \times 10^{-5} \mathrm{~mol} / \mathrm{L}\right)$ at $416 \mathrm{~nm}$ upon addition various amount of $\mathrm{Zn}^{2+}, \mathrm{Hg}^{2+}, \mathrm{Cu}^{2+}$ in $\mathrm{CH}_{3} \mathrm{CN}-\mathrm{H}_{2} \mathrm{O}(V: V=90: 10)$ solution

表 1 受体分子 $1 / 2$ 与不同金属离子络合参数

Table 1 The binding parameters between $\mathbf{1} / \mathbf{2}$ and metal ions

\begin{tabular}{llll}
\hline $\mathbf{1} / \mathbf{2}-\mathrm{M}^{2+}$ & Binding constant & $\begin{array}{l}\text { Limit of } \\
\text { detection }\end{array}$ & $\begin{array}{l}\text { Saturated } \\
\text { equivalent/equiv. }\end{array}$ \\
\hline $\mathbf{1}-\mathrm{Hg}^{2+}$ & $2.03 \times 10^{3}$ & $3.52 \times 10^{-4}$ & 250 \\
$\mathbf{2}-\mathrm{Zn}^{2+}$ & $6.73 \times 10^{3}$ & $8.4 \times 10^{-7}$ & 5 \\
$\mathbf{2}-\mathrm{Hg}^{2+}$ & $1.40 \times 10^{4}$ & $4.1 \times 10^{-7}$ & 5 \\
$\mathbf{2}-\mathrm{Cu}^{2+}$ & $1.97 \times 10^{3}$ & $1.2 \times 10^{-5}$ & 5 \\
\hline
\end{tabular}

分别为 $8.4 \times 10^{-7}, 4.1 \times 10^{-7}$ 和 $1.2 \times 10^{-5} \mathrm{~mol} / \mathrm{L}$, 表明 2 对这些离子苂光识别能力顺序为 $\mathrm{Cu}^{2+}>\mathrm{Zn}^{2+}>$ $\mathrm{Hg}^{2+}$. 同时根据滴定数据可发现, 虽然环受体 1 较非环 受体 2 有更好的苂光识别选择性，而其苂光识别灵敏性 却有很大的减弱，就可能归因于受体分子 $\mathbf{1}$ 中大的刚性 环结构，使得其不能同时与 $\mathbf{1}$ 中的 $\mathrm{N}, \mathrm{O}$ 配位原子发生强 的配位键合.

笔者同时测定了受体环状 $\mathbf{1}$ 在其他竞争性金属离子 存在时对 $\mathrm{Hg}^{2+}$ 的苂光响应. 从图 4 可看出，在其他金属 离子存在时, 受体 $\mathbf{1}$ 对 $\mathrm{Hg}^{2+}$ 仍然有很好的识别能力, 可 用于 $\mathrm{Hg}^{2+}$ 选择性识别的荧光探针. 同时, 探针 $\mathbf{1}$ 在不同 $\mathrm{pH}$ 值时对 $\mathrm{Hg}^{2+}$ 也进行了探讨, 受体分子在 $\mathrm{pH}$ 值 4 9 范围内对 $\mathrm{Hg}^{2+}$ 都有很好的识别能力.

文章同时测定 $1 / 2$ 中滴加不同金属离子时紫外光谱 变化. 不同于荧光光谱, 滴加 $\mathrm{Zn}^{2+}, \mathrm{Hg}^{2+}, \mathrm{Cu}^{2+}$ 及其他金 属离子时, 受体分子 $1 / 2$ 的吸收光谱变化不大(图 5), 表 明受体分子与金属络合后对其基态电子光谱影响并不 大.

\section{3 识别机理研究}

受体分子 $1 / 2$ 对金属离子苂光响应可归因于受体分 子中 $\mathrm{O}$ 及 $\mathrm{N}$ 原子与金属离子配位的结果. 由于 $\mathbf{1}$ 的溶解 性差，本文以 2 为例通过 ${ }^{1} \mathrm{H} N M R$ 滴定来证实这一设想. 


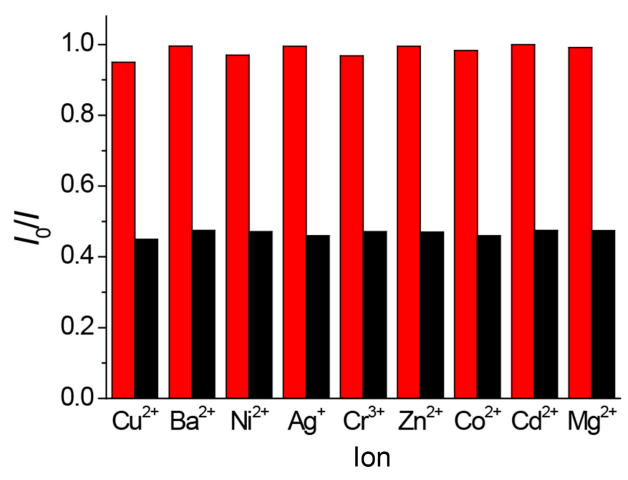

图 $4 \mathrm{CH}_{3} \mathrm{CN}-\mathrm{H}_{2} \mathrm{O}(V: V=90: 10)$ 溶液中受体 $1\left(2.0 \times 10^{-5}\right.$ $\mathrm{mol} / \mathrm{L}$ )滴加 20 equiv. 其他金属离子再滴加 20 equiv. $\mathrm{Hg}^{2+}$ 在 423 $\mathrm{nm}$ 荧光强度变化柱状图

Figure 4 Bar graph of $1\left(2.0 \times 10^{-5} \mathrm{~mol} / \mathrm{L}\right)$ at $423 \mathrm{~nm}$ upon addition 20 equiv. other metal ions and then 20 equiv. $\mathrm{Hg}^{2+}$ in $\mathrm{CH}_{3} \mathrm{CN}-\mathrm{H}_{2} \mathrm{O}(V: V=90: 10)$ solution

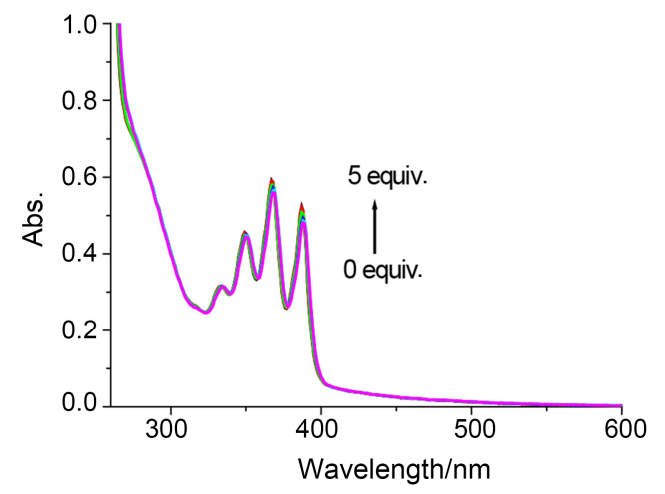

图 5 在 $\mathrm{CH}_{3} \mathrm{CN}-\mathrm{H}_{2} \mathrm{O}(V: V=90: 10)$ 溶液中滴加 $\mathrm{Zn}^{2+}$ 化合物 $2\left(2.0 \times 10^{-5} \mathrm{~mol} / \mathrm{L}\right)$ 的紫外光谱变化图

Figure 5 UV-Vis spectra of $2\left(2.0 \times 10^{-5} \mathrm{~mol} / \mathrm{L}\right)$ upon addition various amount of $\mathrm{Zn}^{2+}$ in $\mathrm{CH}_{3} \mathrm{CN}-\mathrm{H}_{2} \mathrm{O}(V: V=90: 10)$ solution

图 6 为在 $\mathrm{CD}_{3} \mathrm{CN} / \mathrm{CDCl}_{3}(V: V=8: 2)$ 溶液中受体分子 2 中滴加不同当量 $\mathrm{Hg}^{2+}$ 时 ${ }^{1} \mathrm{H} \mathrm{NMR}$ 变化图. 随着 $\mathrm{Hg}^{2+}$ 的 加入, 受体分子三唑环上的氢 $\mathrm{H}(1)$ 化学位移发生了从 $\delta$ 7.17 到 7.32 的低场移动(加入 3 equiv. $\mathrm{Hg}^{2+}$ ), 同时烷氧 键邻位亚甲基上的质子 $\mathrm{H}(3)$ 发生了高场移动 $(\Delta \delta=0.05)$ 及从单峰裂分为四重峰的变化. 这表明受体分子中 $\mathrm{O}$ 及 $\mathrm{N}$ 原子可能发生了金属离子配位.

DFT 计算结果也进一步证明了受体分子中 $\mathrm{N}$ 及 $\mathrm{O}$ 原子参与金属配位的合理性. 配合物 $1-\mathrm{Hg}^{2+}$ 与 $2-\mathrm{Hg}^{2+}$ 的 DFT 计算最优构型见图 7. 从图中看出, 受体分子 $\mathbf{1}$ 与 2 中的三唑环上的 3-位 $\mathrm{N}$ 原子及醚氧键中的 $\mathrm{O}$ 原子与中 心原子 $\mathrm{Hg}^{2+}$ 参与了的配位. 从键长数据可看出, 两受体 分子中 $\mathrm{N}$ 原子的配位能力强于 $\mathrm{O}$ 原子. 同时从图中还可 看出, 虽然 1 与 2 中配位原子与中心离子配位键长相差 不大，但 $1-\mathrm{Hg}^{2+}$ 中金属离子 $\mathrm{Hg}^{2+}$ 位于环的侧面(图 7b),
2- $\mathrm{Hg}^{2+}$ 则位于受体分子平面腔孔中。这种侧面配位 $\left(1-\mathrm{Hg}^{2+}\right)$ 与平面配位差异可解释受体 1 较 2 更弱的与 $\mathrm{Hg}^{2+}$ 络合能力结果.

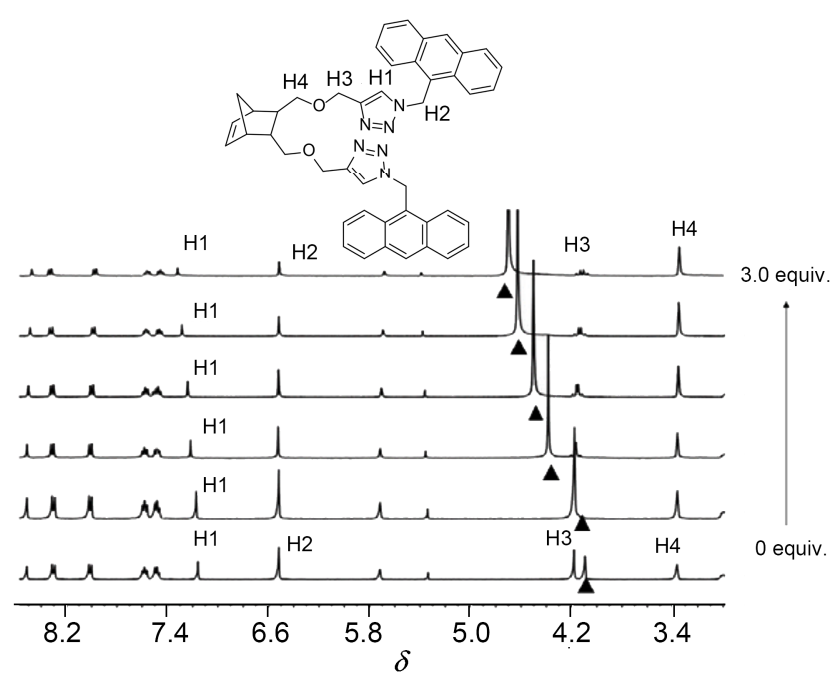

图 $6 \mathrm{CD}_{3} \mathrm{CN} / \mathrm{CDCl}_{3}(V: V=8: 2)$ 溶液中化合物 2 对 $\mathrm{Hg}^{2+}$ 的 核磁滴定图

Figure $6{ }^{1} \mathrm{H}$ NMR spectrum of 2 upon addition of $\mathrm{Hg}\left(\mathrm{ClO}_{4}\right)_{2}$ in $\mathrm{CD}_{3} \mathrm{CN} / \mathrm{CDCl}_{3}(V: V=8: 2)$ solution

$\Delta$ represents the residual water peak in system a)
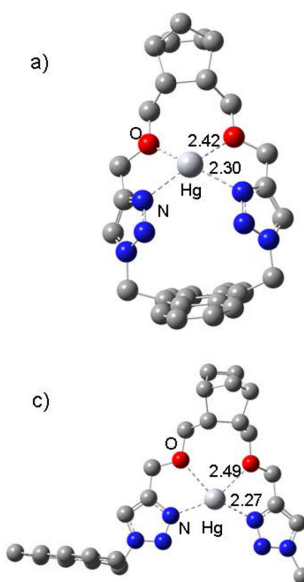

b)

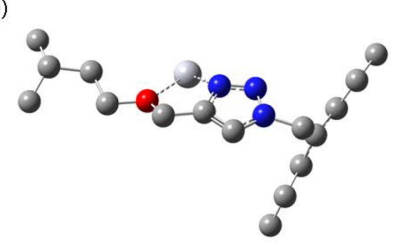

d)

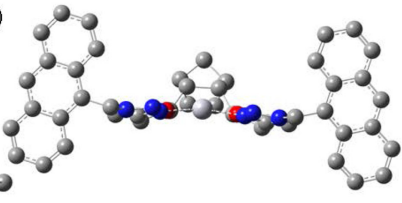

图 $71-\mathrm{Hg}^{2+}$ 与 $2-\mathrm{Hg}^{2+}$ 络合 DFT 计算最优构型

Figure 7 Optimized structures of $\mathbf{1}-\mathrm{Hg}^{2+}$ and $2-\mathrm{Hg}^{2+}$ complexes by DFT calculations

The $\mathrm{a}$ and $\mathrm{c}$ present top views, and $\mathrm{b}$ and $\mathrm{d}$ present the side views. The hydrogen atoms and anions are omitted for clarity

\section{2 结论}

通过点击反应合成了一种以降冰片烯为骨架含葱 荧光团非环及环状双三唑化合物 $\mathbf{1}$ 和 $\mathbf{2}$, 并研究了其对 金属离子荧光识别能力. 在 $\mathrm{CH}_{3} \mathrm{CN}-\mathrm{H}_{2} \mathrm{O}(V: V=90$ : 10)中, 荧光光谱表明环受体 $\mathbf{1}$ 仅对 $\mathrm{Hg}^{2+}$ 有荧光淬灭响 应, 而 2 对 $\mathrm{Zn}^{2+}, \mathrm{Hg}^{2+}$ 与 $\mathrm{Cu}^{2+}$ 都有很好的苂光识别能力, 
其荧光识别能力顺序为 $\mathrm{Cu}^{2+}>\mathrm{Zn}^{2+}>\mathrm{Hg}^{2+}$. 同时研究 发现虽然 1 较 2 具有更好的金属离子识别选择性, 但其 苂光识别灵敏度有很大减弱. ${ }^{1} \mathrm{H}$ NMR 滴定及 DFT 计算 表明受体分子受体分子中的三唑环上的 3 位 $\mathrm{N}$ 原子及醚 氧基团中的 $\mathrm{O}$ 原子参与了金属配位. 同时 DFT 计算表 明非环受体 2 较 1 有更好同 $\mathrm{Hg}^{2+}$ 尺寸匹配, 导致其有更 好的离子识别灵敏性. 目前虽然大量文献报道环状受体 分子由于好的预组织性及刚性结构较非环受体有更好 的客体分子选择性与灵敏性识别 ${ }^{[32]}$, 本文的结果表明 主客体间空间尺寸匹配性常有更大的影响. 本文的研究 为设计高选择性与灵敏度的环状受体具有一定的参考 价值.

\section{3 实验部分}

\section{1 仪器与试剂}

化合物 3, 4 和 5 按文献方法合成, 其它试剂及药品 (分析纯)购于国药集团化学试剂公司; 无水溶剂按文献 方法纯化制备, 其他试剂均为分析纯, 使用前未作进一 步纯化.

核磁共振仪(德国 Bruker 公司, Bruker Vance 400 $\mathrm{MHz}, \mathrm{TMS}$ 为内标)、紫外可见光谱(HITACHI U-3010 型 紫外可见分光光度计)、苂光光谱(Hitachi F-4600 型苂光 光谱仪). 滴定所用金属离子 $\mathrm{Na}^{+}, \mathrm{Mg}^{2+}, \mathrm{Ba}^{2+}, \mathrm{Hg}^{2+}$, $\mathrm{Cu}^{2+}, \mathrm{Cr}^{3+}, \mathrm{Zn}^{2+}, \mathrm{Fe}^{2+}, \mathrm{Ni}^{2+}, \mathrm{Co}^{2+}, \mathrm{Cd}^{2+}, \mathrm{Ag}^{+}$等)均为高 氯酸盐.

\section{2 实验方法}

3.2.1 ( $\left(1^{4} Z, 5^{4} Z, 9^{1} R, 9^{3} S, 9^{4} S\right)-1^{1} H, 5^{1} H-7,11-$ 二氧 $-1,5$ $(1,4)$-二三唑-3(9,10)-葱-9(2,3)-二环[2.2.1]庚烷环二十 烷 $-9^{5}-$ 烯 $(1)$ 的合成

在氮气保护下, 将 $1.5 \mathrm{~mL}$ 的 1,8-二氮杂二环 [5.4.0] 十一碳-7-烯(DBU, $10 \mathrm{mmol}$ )及 $28.5 \mathrm{mg} \mathrm{CuI} \mathrm{(0.015}$ $\mathrm{mmol}$ )加入到 $200 \mathrm{ml}$ 除水甲苯中, 并将溶液升温至 $70{ }^{\circ} \mathrm{C}$. 同时化合物 3 (115 mg, $\left.0.5 \mathrm{mmol}\right)$ 和 4 (144 mg, $0.5 \mathrm{mmol}$ )溶于 $100 \mathrm{~mL}$ 除水甲苯中, 并缓慢滴加到上述 溶液. 滴加约 $8 \mathrm{~h}$, 滴加完毕后, 继续反应 $2 \mathrm{~h}$. 减压蒸馏 除去溶剂, 残留物用 $\mathrm{CH}_{2} \mathrm{Cl}_{2} / \mathrm{MeOH}(V: V=98: 2)$ 过柱, 得到 $102 \mathrm{mg}$ (0.18 mmol)白色固体 1, 收率 39\%. m.p. $198 \sim 199{ }^{\circ} \mathrm{C} ;{ }^{1} \mathrm{H}$ NMR (400 MHz, DMSO- $d_{6}$ ) $\delta: 8.77$ (s, 2H), 8.44 (s, 2H), 7.74 (s, 2H), 7.59 (s, 2H), 7.02 (s, 2H), 6.77 (q, $J=15.4 \mathrm{~Hz}, 3 \mathrm{H}), 5.73(\mathrm{~s}, 1 \mathrm{H}), 4.46(\mathrm{~d}, J=13.8$ $\mathrm{Hz}, 2 \mathrm{H}), 4.27$ (s, 2H), 3.90 (d, $J=13.3 \mathrm{~Hz}, 2 \mathrm{H}), 2.35$ (s, 2H), 1.91 (s, 2H), $1.61(\mathrm{~s}, 2 \mathrm{H}), 1.21(\mathrm{~s}, 2 \mathrm{H}), 0.94(\mathrm{~s}, 1 \mathrm{H})$, $0.86(\mathrm{~s}, 1 \mathrm{H}) ;{ }^{13} \mathrm{C}$ NMR (101 MHz, DMSO- $\left.d_{6}\right) \delta$ : 143.6, $134.5,130.7,130.17,128.6,127.6,125.2,124.8,123.3$, $67.1,62.8,45.7,44.4,40.5,40.1,39.9,39.8,39.5,39.3 \sim$ $38.9(\mathrm{~m})$; ESI-MS m/z: $520.25(\mathrm{M}+1)^{+}$. Anal. calcd for $\mathrm{C}_{31} \mathrm{H}_{30} \mathrm{~N}_{6} \mathrm{O}_{2}$ : C 71.79, H 5.83, N 16.20; found $\mathrm{C} 71.65, \mathrm{H}$ $5.85, \mathrm{~N} 16.32$.

$3.2 .2(1 R, 4 S, 5 R)-5,6$-二\{\{[1-(葱-9-亚甲基)-1 $H-1,2,3-$ 三唑-4-亚]甲氧基\}甲基 $\}$ 二环 [2.2.1]庚烷-2-烯(2)的合 成

在氮气保护下, 将 $115 \mathrm{mg}(0.5 \mathrm{mmol})$ 化合物 3、280 $\mathrm{mg}(1.2 \mathrm{mmol})$ 化合物 $5 、 25 \mathrm{mg}$ 五水硫酸铜、20 mg 抗 坏血酸钠溶于 $10 \mathrm{~mL} \mathrm{DMF}$ 中，反应混合液室温下反应 4 h. 待反应结束后, 在反应液中加入氯化钠水溶液, 过 滤, 取滤渣. 干燥后粗产物用二氯甲烷-甲醇的混合液 体 $(V: V=98: 2)$ 过柱, 减压蒸馏, 得到 $284 \mathrm{mg}$ 白色固 体 2, 产率 $80 \%$. m.p. $145 \sim 146{ }^{\circ} \mathrm{C} ;{ }^{1} \mathrm{H}$ NMR (400 MHz, $\left.\mathrm{CDCl}_{3}\right) \delta: 8.51(\mathrm{~s}, 2 \mathrm{H}), 8.31(\mathrm{~d}, J=8.8 \mathrm{~Hz}, 4 \mathrm{H}), 8.01(\mathrm{~d}$, $J=8.0 \mathrm{~Hz}, 4 \mathrm{H}), 7.59 \sim 7.56(\mathrm{~m}, 4 \mathrm{H}), 7.50 \sim 7.46(\mathrm{~m}, 4 \mathrm{H})$, $7.10(\mathrm{~s}, 2 \mathrm{H}), 6.51(\mathrm{~s}, 4 \mathrm{H}), 5.73(\mathrm{~s}, 2 \mathrm{H}), 5.30(\mathrm{~s}, 2 \mathrm{H})$, $4.25 \sim 4.18(\mathrm{~m}, 4 \mathrm{H}), 4.25 \sim 4.18(\mathrm{~m}, 4 \mathrm{H}), 3.03(\mathrm{t}, J=13.2$ $\mathrm{Hz}, 2 \mathrm{H}), 2.81$ (t, $J=15.4 \mathrm{~Hz}, 2 \mathrm{H}), 2.66(\mathrm{~s}, 2 \mathrm{H}), 2.18$ (s, $4 \mathrm{H}), 1.61 \sim 1.58(\mathrm{~m}, 1 \mathrm{H}), 1.27(\mathrm{~d}, J=16.2 \mathrm{~Hz}, 1 \mathrm{H}) ;{ }^{13} \mathrm{C}$ NMR (100 MHz, $\left.\mathrm{CDCl}_{3}\right) \delta: 31.0,41.2,45.3,46.3,48.8$, $53.5,64.1,70.5,122.0,123.0,123.8,125.4,127.6,129.4$, $129.8,129.8,130.8,131.4,135.0,145.3$; ESI-MS $m / z$ : $698.22(\mathrm{M}+1)^{+}$. Anal. calcd for $\mathrm{C}_{45} \mathrm{H}_{40} \mathrm{~N}_{6} \mathrm{O}_{2}$ : C 77.56, $\mathrm{H}$ 5.79, N 12.06; found C 77.72, H 5.83, N 12.14.

\section{3 紫外及荧光光谱滴定实验}

紫外及苂光光谱测定受体分子浓度为 $2 \times 10^{-5} \mathrm{~mol} /$ $\mathrm{L}$ 乙腈-水 $(V: V=90: 10)$ 溶液, 金属离子配制成 $2 \times$ $10^{-3} \mathrm{~mol} / \mathrm{L}$ 溶液. 苂光光谱测定时激发波长为 $360 \mathrm{~nm}$. 测 试时用移液枪向 1 和 2 中滴加高氯酸盐的阳离子, 观察 受体分子与阳离子结合后紫外及苂光光谱变化.

\section{$3.4{ }^{1} \mathrm{H}$ NMR 滴定测试}

Bruker Vance $400 \mathrm{MHz}$ 核磁共振仪, $\mathrm{CD}_{3} \mathrm{CN}$ 溶液中 化合物 $2\left(2.0 \times 10^{-4} \mathrm{~mol} / \mathrm{L}\right)$ 中利用累积滴加法逐次加入 高氯酸盐的阳离子, 观察受体分子 $\mathbf{2}$ 与 $\mathrm{Hg}^{2+}$ 结合后活泼 氢化学位移的变化.

\section{5 量子化学结构计算}

采用高斯 09 的 B3LYP/6-31G* $(\mathrm{d}, \mathrm{p})$ 方法, 计算目标 化合物 1,2 与阳离子作用后的结构模型.

辅助材料(Supporting Information) 化合物 1 和 2 与金 属离子的紫外光谱图, ${ }^{1} \mathrm{H} \mathrm{NMR} 、{ }^{13} \mathrm{C} N \mathrm{NMR}$ 及 ESI-MS. 这 些材料可以免费从本刊网站(http://sioc-journal.cn/)上下 载. 


\section{References}

[1] Masayuki, M.; Michael, M.; Peter, W.; Richard, A.; Kim, D. Org. Lett. 2005, 7, 4943.

[2] Kallol, B.; Anand, K.; Moupriya, N.; Soumen, B. Anal. Chem. 2014, 86, 2740.

[3] Zhang, X.; Guo, C.; Li, Z.; Shen, G.; Yu, R. Anal. Chem. 2002, 74, 821 .

[4] Seraphine, V.; Hasan, A.; Murat, S.; Jun, Y.; Chuan, H. J. Am. Chem. Soc. 2010, 132, 2567

[5] Wu, L.; Guo, Q.; Liu, Y.; Sun, Q. Anal. Chem. 2015, 87, 5318.

[6] Koushik, G.; Sudipto, D.; Shibashis, H.; Aradhita, B.; Corrado, R.; Partha, R. J. Mol. Struct. 2016, 325, 1118.

[7] Mehdi, A.; Roghayeh, S.; Soraia, M. Sens. Actuators, B 2016, $233,355$.

[8] Ding, Y.; Zhu, W.-H.; Xie, Y. Chem. Rev. 2017, 117, 2203.

[9] Ding, Y.; Li, X.; Li, T.; Zhu, W.; Xie, Y. J. Org. Chem. 2013, $78,5328$.

[10] Xie, Y.; Wei, P.; Li, X.; Hong, T.; Zhang, K.; Furuta, H. J. Am. Chem. Soc. 2013, 135, 19119.

[11] Ding, Y.; Tang, Y.; Zhu, W.; Xie, Y. Chem. Soc. Rev. 2015, 44, 1101.

[12] Tang, Y.; Ding, Y.; Li, X.; Ågren, H.; Li, T.; Zhang, W.; Xie, Y. Sen. Actuators, B 2015, 206, 291.

[13] Martınez-Manez, R.; Sancenon, F. Chem. Rev. 2003, 103, 4419.

[14] Kolb, H.; Finn, M.; Sharpless, B. Angew. Chem., Int. Ed. 2001, 40, 2004.

[15] Lau, Y. H.; Rutledge, P. J.; Watkinson, M.; Todd, M. H.
Chem. Soc. Rev. 2011, 40, 2848.

[16] Ge, J.; Liu, Z.; Cao, Q.; Chen, Y ; Zhu, J. Chem. Asian J. 2016, $11,687$.

[17] Dai, B.; Cao, Q.; Wang, L.; Wang, Z.; Yang, Z. Inorg. Chim. Acta 2014, 423, 163.

[18] Zhu, J.; Fan, X.; Cao, Q. Inorg. Chim. Acta 2016, 451, 111.

[19] Wang, Z ; Palacios, M.; Pavel, A. Anal. Chem. 2008, 80, 7451.

[20] ]Wu, Y.; Tan, Y.; Wu, J.; Chen, S.; Chen, Y.; Zhou, X.; Jiang, Y.; Tan, C. ACS Appl. Mater. Interfaces 2015, 7, 6882.

[21] Wang, Z.; Dai, B.; Qiu, J.; Cao, Q.; Ge, J. Chin. J. Org. Chem. 2015, 35, 2383 (in Chinese).

(王智成, 戴博娜, 丘继芳, 曹迁永, 葛金柱, 有机化学, 2015, 35, 2383.)

[22] Emiliano, T.; Arna, K.; Lisa, M.; Matthew H. T.; Michael, W. Inorg. Chem. 2009, 48, 319.

[23] Sui, B.; Kim, B.; Zhang, Y.; Frazer, A.; Belfield, K. ACS Appl. Mater. Inter. 2013, 5, 2920.

[24] Maity, D.; Govindaraju, T. Inorg. Chem. 2010, 49, 7229

[25] Xu, K.; Chen, Z.; Zhou, L.; Zheng, O.; Wu, X.; Guo, L.; Qiu, B.; Lin, Z.; Chen, G. Anal. Chem. 2015, 87, 816.

[26] Banti, D.; North, M. Tetrahedron Lett. 2002, 43, 1561.

[27] Zhou, L.; Sun, Q. Chem. Commun. 2015, 51, 16767.

[28] Reuter, R.; Wegner, H. Chem. Commun. 2013, 49, 146.

[29] Kumari, A.; Swamy, K. J. Org. Chem. 2016, 81, 1425.

[30] Caricato, M.; Olmo, A.; Gargiulli, C.; Gattuso, G.; Pasini, D. Tetrahedron 2012, 68, 7861.

[31] Szejtli, J. Cyclodextrine Technology, Kluwer Academic Publishers, Dodrecht, The Netherlands, 1988.

[32] Hua, Y.; Flood, A. H. Chem. Soc. Rev. 2010, 39, 1262. 